Proyecciones

Vol. $13 N^{\circ} 1$, pp. 35-52 July 1994

liniversidad C'atólica del Norte

Antofagasta - Chile

\title{
INVOLUTIVE CO-DISTRIBUTIONS PRESERVED BY TRANSITIVE FAMILIES OF VECTOR FIELDS*
}

\author{
VÍCTOR AYALA BRAVO
}

Universidad Católica del Norte, Antofagasta-Chile.

\begin{abstract}
This paper deals with integrability conditions of involutive co-distributions defined on the co-tangent bundle of a differentiable manifold $M$. Via Frobenius 's integrability theorem, the analysis is aimed at the search of conditions so that this type of co-distributions be preserved by transitive families of vector ficlds in $M$. We rely on the work of Lobry, Sussmann, Matsuda and Stefan. The type of situation studicd comes up naturally in weak-obscruability problcms and weakly-minimal realizations of arbitrary control systems.
\end{abstract}

\section{Keywords}

Co-distributions, transitivity, co-distributions preserved by families of vector ficlds, integrability.

*Supported by Proyecto FONDECYT No 92-0185 and Proyecto D.G.I.C.T. U.C.N. Contro labilidad de Grupos Nilpotentes. 


\section{INTRODIITION}

Let $M$ a differentiable manifold and $\theta$ a co-distribution on the co-tangent bundle $T^{*} M$, that is. for each $x \in M, \theta(x)$ is a vector subspace of the dual of the tangent space to $M$ in $x$. Frobenius's throrem ensures the integrability of $\theta$ if this codistribution is involutive and regular, [10]. In this paper, we are interested in finding conditions that warrant regularity of the involutive co-distribution. As we shall see, the type of situation to be analysed appears in the study of weakobservability and weakly-minimal realization of control systems.

A control systern $\Sigma$ is determined by the following data:

$$
\Sigma=\left(M, D, R^{s}, h\right)
$$

where $\mathrm{M}$ is a differentiable nanifold, $D \subset X(M)$ is a family of vector fields in $M$, (strategies) and $h: M \longrightarrow R^{s}$ is a differentiable function.

Let's denote by $X_{t}$ the flow associated to the vector field $X$. Then $D$ induces a pseudo-group:

$$
G_{z}=\left\{X_{t_{1}}^{1} \circ X_{t_{2}}^{2} \circ \ldots \circ X_{t_{k}}^{k} \mid X^{j} \in D, t_{j} \in \mathbb{R}\right\}
$$

and a semi-pseudo-group

$$
S_{\Sigma}=\left\{X_{t_{1}}^{1} \circ X_{t_{2}}^{2} \circ \ldots \circ X_{t_{k}}^{k} \mid X^{j} \in D, t_{j} \geq 0\right\}
$$

of local diffeomorphisms on $M$.

Gs acts over $M$ on such a way that the orbits

$$
G_{\Sigma}(x)=\left\{\rho(x) \mid \rho \in G_{\Sigma}\right\}, x \in M
$$

form a foliation with singularities, [7]. $\Sigma$ is said to be transitive if there is a single orbit and in this case, for each $x \in M, C_{\Sigma}(x)=M$.

Each orbit $G_{\Sigma}(x)$ has a structure of a differentiable manifold, however the topology can be strictly finer than the induced topology. It is possible to restrict $\Sigma$ to each orbit $\operatorname{Gr}_{\Sigma}(x)$, thus obtaining a natural environment of the system for the initial condition $x,[9]$. Particularly, it is always possible to consider $\Sigma$ as being transitive (on its orbits).

The accessibility set of $\Sigma$ from the point $\mathrm{x}$ is given by its positive orbit under $\Sigma$, that is:

$$
S_{\Sigma}(x)=\left\{\rho(x) \mid \rho \in S_{\Sigma}\right\} \text {. }
$$

If $S_{\Sigma}(x)=M$, we say that the system $\Sigma$ is controllable from $x$. Two states $x, y \in M$ are said to be undistinguishable (by $\Sigma$ ) if

$$
h \circ \rho(x)=h \circ \rho(y), \forall \rho \in S_{\Sigma} .
$$

$\Xi$ is said to be observable if there are no two undistinguishable states in $M$ and weakly-observable in $x \in M$ if there is a neighborhood $U$ of $x$ such that for each 
$y \in I^{\prime}, x$ and $y$ are not undistinguishable, i.e., the family of loc al diffeomorphisms Siv restricted to $U$, separates $x$ from any point $y \in U$.

A minimal realization for $\Sigma$ is a transitive and observable system

$$
\Sigma_{1}=\left(M_{1}, D_{1}, R^{s}, h_{1}\right)
$$

and a submersion $\pi: M \longrightarrow M_{1}$ such that : each vector field $X \in D$ is projectable on $M_{1}$ via the differential $d \pi=\pi_{*}, \pi_{*}(D)=D_{1}$, and $h=h_{1} \circ \pi$.

Two states $x, y \in M$ are said to be quasi-undistinguishable (by $\Sigma$ ) if :

$$
h \circ \rho(x)=h \circ \rho(y), \quad \forall \rho \in G_{\Sigma} .
$$

The concepts of quasi-observability and weak-observability for minimal realization are defined in an analogous way.

The study of the olsservability of $\Sigma$ naturally leads to the analysis of the curves $h_{i} \circ \rho(x), 1 \leq i \leq s, \rho \in S_{\Sigma}$.

Hermann and Krener, with the purpose of studying sufficient conditions of weak-observability, introduced in [4] the co-distribution defined by :

$$
\Lambda=\operatorname{Span}\left\{L_{x^{1}} \circ L_{x^{2}} \circ \ldots \circ L_{x^{r}}\left(d h_{j}\right) \mid X^{j} \in D, 1 \leq i \leq s\right\}
$$

where $L_{x}\left(h_{i}\right)(\cdot)=d h_{i(\cdot)} X(\cdot), 1 \leq i \leq s$, is Lie's derivative of $h_{i}$ in the direction of the vector field $X$. Since this derivative commutes with the exterior derivative [10], that is $d L_{x}(f)=L_{x}(d f)$ for any function $f: \operatorname{dom}(f) \subset M \longrightarrow R, \Lambda$ is, to a certain extent, an infinitesimal measure of $h$ with respect to all possible concatenations of the system strategies.

$\ln [4]$ it is proved the so-called weak-observability rank condition:

$\operatorname{dim} \Lambda(x)=\operatorname{dim} M \Longrightarrow \Sigma$ is weakly observable in $x$.

In [3] Basto Gonçalvez studies the problem of the quasi-observability of systems and defines $\nabla$ as the smallest $\Sigma$ invariant co-distribution containing the $d h_{i}, 1 \leq$ $i \leq s$, and builds quasi-minimal realization of $\Sigma$, through the integral manifolds of the regular co-distribution $\nabla$.

Ayala and San Martin introduce in [1] the co-distribution

$$
\nabla^{+}=\operatorname{Span}\left\{\rho^{*}\left(d h_{i}\right) \mid \rho \in S_{\Sigma}, 1 \leq i \leq s\right\}
$$


where the pull-back is defined by $\rho^{*}\left(d h_{i}\right)=d\left(h_{i} \circ \rho\right)$, obtaining weakly-minimal realization of controllable systems in the process of building minimal realization of this type of systems.

As we shall see for transitive systems, $\nabla$ will always be integrable, however this may not true for $\Lambda$ and $\nabla^{+}$. On the other hand, $\Lambda \subset \nabla^{+} \subset \nabla$ and since $\Lambda$ is in general less difficult to build, it is convenient to specify the situations such that $\Lambda=\nabla^{+}, \Lambda=\nabla^{+}=\nabla$. Under these circumstances, the study of the observability of control systems leads to the search of integrability conditions of involutive codistributions in the sense of differential ideals, in fact, $\nabla^{+}$and $\nabla$ are generated by families of exact 1-forms. Consequently, the condition of regularity (i.e., that the dimension of this type of co-distributions remains constant on $M$ ) is sufficient to determine integrability in the classical sense by a direct application of Frobenius theorem [10].

For this purpose, we rely on the techniques used by Lobry [5], Sussmann [9], Matsuda [6] and Stefan [8] in relation to the study of the integrability of distributions on the tangent bundle $T M$.

\section{CO-DISTRIBUTIONS}

Let us begin with some basic concepts. For each differentiable manifold $M$ and for each $x$ in $M, T_{x}^{*} M$ will denote the dual of the vector space $T_{x} M$, that is

$$
T_{x}^{*} M=\left\{\lambda: T_{x} M \longrightarrow \operatorname{R} \mid \lambda \text { linear }\right\} .
$$

The co-tangent bundle on $M$ is by definition the manifold

$$
T^{*} M=\bigcup_{x \in M} T_{x}^{*} M
$$

A co-distribution $\theta$ in $M$ is a map such that for each $x \in M, \theta(x)$ is a vector sub-space of $T_{x}^{*} M . \theta$ will be said to be regular if $\operatorname{dim} \theta(x)$ is independent from $x$ in $M$.

An immersion $(N, i)$ is said to be a (maximal) integral manifold of a codistribution $\theta$ if for each $x \in N$ :

a) $\theta(i(x))\left(\left(d i_{x}\left(T_{x} N\right)\right)=0\right.$,

b) $\operatorname{dim}(N)=\operatorname{dim}(M)-\operatorname{dim} \theta(i(x))$.

$\theta$ is integrable in $x[\operatorname{in} M]$, if there is an integral manifold of $\theta$ containing $x$, denoted $\operatorname{In}_{\theta}(x)$ [for each $\left.x \in M\right]$.

Let us look at these concepts under the light of the co-distributions $\Lambda, \nabla^{+}$and $\Gamma$.

Examples 2.0. 
1. Let $\Sigma=\left(\operatorname{IR}, D=\left\{u \frac{d}{d x} \mid u \in \mathbb{R}\right\}, \mathbb{R}^{2}, h=(\cos x\right.$, sen $\left.x)\right)$. Then,

$$
\Lambda(x)=\operatorname{Span}\{\operatorname{sen} x d x, \cos x d x\}=T_{x}^{*} \mathrm{IR} .
$$

But $T_{x}^{*}$ IR can be nullified only at the origin $x$. Thus, the integral manifold of $\Lambda$ at $x \in \mathbb{R}$ is $\operatorname{In}_{\Lambda}(x)=\{x\}$.

Evidently, $i$ is the inclusion. Now, since $\Lambda \subset \nabla^{+} \subset \nabla$ we have that $\Lambda=\nabla^{+}=$ $\Gamma$

Let $\Sigma=\left(\mathrm{IR}^{2}, D=\left\{u \frac{\partial}{\partial x} \mid u \in \mathrm{IR}\right\}, \mathrm{IR}, h\right)$ be a control system such that

$$
h(x, y)=\int_{-\infty}^{x} f(t) d t
$$

where $f$ is a function of class $C^{\infty}$ that is zero in $(-\infty, 0]$ and strictly increasing on $(0,+\infty)$.

For each $w=(x, y) \in \mathbb{R}^{2}$ we have

$$
\Lambda(w)=\operatorname{Span}\left\{f^{(k)}(x) d x \mid K \geq 0\right\} .
$$

Then,

$$
\Lambda(w)=\left\{\begin{array}{cc}
\operatorname{Span}\{d x\} & \text { if } x>0 \\
0 & \text { if } x \leq 0
\end{array}\right.
$$

Thus, if $\pi_{1}$ denotes the projection on the $x$ axis we have:

a) $J=\left\{w \in \mathbb{R}^{2} \mid \pi_{1}(w)<0\right\}$ is the integral manifold of $\Lambda$ through each $w \in J$.

b) $\operatorname{Span}\left\{\left.\frac{\partial}{\partial y}\right|_{w}\right\}$ is the integral manifold of $\Lambda$ containing $w$ for each $w$ satisfying $\pi_{1}(w)>0$.

c) There are no integral manifolds of $\Lambda$ containing elements with null first coordinate. In fact, since all the manifolds considered are boundaryless, each integral manifolds of $\Lambda$ "passing" over these points will have to "go through" the $y$ axis. This situation is not possible because of the difference of the dimension of $\Lambda$ in any neighborhood of this type of state.

The integrability of $\nabla^{+}$comes from the equality

$$
\nabla^{+}(x, y)=\operatorname{Span}\left\{f^{(k)}(x+t) d x \mid t>0\right\}=\operatorname{Span}\{d x\}
$$


In particular, $\nabla^{+}$is regular and for each $w=(x, y) \in \mathbb{R}^{2}$ we have $\operatorname{In}_{\nabla+}(x, y)=$ Span $\left\{\left.\frac{\partial}{\partial y}\right|_{w}\right\}$. Besides, we can observe that in this case $\Lambda \subset \nabla^{+}=\nabla$.

Definition 2.1. Let $X \in X(M)$ and $\theta$ a co-distribution in $M$. We say that $X$ preserves $\theta$ if for each $x \in \operatorname{dom}(X)$ :

$$
X_{t}^{*} \theta\left(X_{t}(x)\right) \subset \theta(x), \text { for each possible } t \in \mathbb{R} \text {. }
$$

$F \subset X(M)$ preserves $\theta$ if each $X \in F$ preserves $\theta$.

\section{Remarks 2.2.}

1. If $\mathrm{X}$ preserves $\theta$ then, for each $x \in \operatorname{dom}(X)$

$$
X_{t}^{*} \theta\left(X_{t}(x)\right)=\theta(x) \text { for each possible } t \in \mathbb{R} \text {. }
$$

2. Let $F$ be a transitive family of vector fields on $M$, that is, the pseudo-group $G_{F}$ built analogously to pseudo-group $G_{\Sigma}$ for control systems, is such that:

$$
\exists x \in M \text { satisfying } G_{F}(x)=M \text {. }
$$

Let us suppose that $F$ preserves $\theta$, then, according to the remark above, the dimension of $\theta$ is independent from $x \in M$ and thus, $\theta$ is regular. It is important to note here the relevance of the orbit theorem [9]. Indeed, given a control system $\Sigma$ on $M$, the field family $F=D_{\Sigma}$ is transitive over each orbit of $\Sigma$. Particularly, each co-distribution $\theta$ preserved by $D_{\Sigma}$ will be regular.

3. Note that $-\frac{\partial}{\partial x}$ does not preserve $\Lambda=\operatorname{Span}\left\{f^{(k)} d x\right\}$ in example $2.0(2)$.

4. It is well-known that the operator of derivative exterior $d$ satisfies $d^{2}=0$. In particular, the exterior derivative of an exact form $\mathrm{df}$ is null. Remember that the co-distributions introduced in [section 1] were defined by

5.

$$
\begin{gathered}
\Lambda=\operatorname{Span} .\left\{d\left(L_{X^{1}} \circ \ldots \circ L_{X^{r}}\left(h_{i}\right) \mid X^{j} \in D, 1 \leq i \leq s\right\}\right. \\
\nabla^{+}=\operatorname{Span}\left\{d\left(\rho \circ h_{i}\right) \quad \mid \rho \in S_{\Sigma}, 1 \leq i \leq s\right\} \\
\nabla=\operatorname{Span}\left\{d\left(\rho \circ h_{i}\right) \quad \mid \rho \in G_{\Sigma}, 1 \leq i \leq s\right\}
\end{gathered}
$$

consequently,

$$
d(\Lambda)=d\left(\nabla^{+}\right)=d(\nabla)=0 .
$$

In particular, these co-distributions are involutive. So, we must try to find conditions under which this type of co-distributions is preserved by transitive families of vector fields. 
The introduction of the concept that follows is thoroughly justified. Let $w$ a 1 - form in $M$, that is:

$$
w: x \in \operatorname{dom}(w) \subset M \longrightarrow w_{x} \in T_{x}^{*} M
$$

and let $X \in X(M)$. Lie's derivative of $w$ in the direction of the vector field $X$ in $x \in \operatorname{dom}(X) \cap \operatorname{dom}(w)$ is by definition:

$$
\left.L_{X}(w)\right|_{r}(\cdot)=\left.\frac{d}{d t}\right|_{t=0} w_{X t(x)}\left(X_{t}^{*}(x)(\cdot)\right)
$$

Now, let $\theta$ a co-distribution in $M$ parametrized by $\delta=\left(w^{1}, \ldots, w^{r}\right)$ over a "piece of trajectory of $X$ in $x "$, that is,

$$
\exists \varepsilon>0: \theta\left(X_{t}(x)\right)=\operatorname{Span}\left\{w_{X_{t}}^{1}(x), \ldots, w_{X_{t}}^{r}(x)\right\}
$$

for each $t \in(-\varepsilon, \varepsilon)$. Then, by definition, a vector field $X$ preserves $\theta$ if:

$$
X_{t}^{*}\left(\theta\left(X_{t}(x)\right) \subset \theta(x)\right.
$$

equivalently, if the curves

$$
\Psi_{j}(t)=X_{t}^{*} w_{X t}^{j}(x) \in \theta(x), 1 \leq j \leq r .
$$

By derivation, we obtain for each $j$ :

$$
\frac{d}{d t} \Psi_{j}(t)=X_{t}^{*} L_{x}\left(w^{j}\right)\left(X_{t}(x)\right)
$$

In particular, and since for each $j=1, \ldots, r, w_{x}^{j} \in \theta(x)$ we can conclude that a necessary condition for $X$ to preserve $\theta$ is:

$$
\frac{d}{d t} \Psi_{j}(t) \in \theta(x)
$$

and then with $t=0$, we obtain that Lie's derivative of $w^{j}$ in the direction of the vector field $X$ at $x$ must belong to $\theta$, that is

$$
\left.L_{X}\left(w^{j}\right)\right|_{x} \in \theta(x)
$$

for each $x \in \operatorname{dom}(X) \cap \operatorname{dom}\left(w^{j}\right), j=1, \ldots, r$.

However, as shown in example $2.0(2)$, this condition is not sufficient and therefore. if we wish to study integrability via the above notion, we need some additional hypothesis on these derivatives.

Definition 2.3. Let $\theta$ be a co-distribution, 
1. $\theta$ is said to be differentiable at $x$, if there are 1 -forms $w^{1}, \ldots, w^{r}$ differentiable in a neighborhood $I$ in $x$ satisfying

i) $\mathrm{w} \in \theta$ on $\mathrm{U}$

ii) $\theta(\mathrm{x})=\operatorname{Span}\left\{w_{x}^{1}, \ldots, w_{x}^{r}\right\}$.

2. Let $\theta$ be differentiable at $x$, the map

$$
y \in U \cdot \longrightarrow \delta(y): R^{r} \longrightarrow \theta(y) \subset T_{y}^{*} M
$$

defined by

$$
\delta(y)\left(t_{1}, \ldots, t_{r}\right)=\Sigma_{j=1}^{r} t_{j} w_{y}^{j}
$$

is called a parametrization of $\theta$ (centered in $x$ ). We denote $\delta=\left(w^{1}, \ldots, w^{r}\right)$.

3. Let $\delta=\left(w^{1}, \ldots, w^{r}\right)$ a parametrization of $\theta$ centered in $x$. We say that a vector field $X$ in $M$ preserves $\theta$ infinitesimally at $x$ along $\delta$ if :

i) there is an interval $J$ containing the origin such that

$$
t \in J \Longrightarrow \theta\left(X_{t}(x)\right)=\operatorname{Span}\left\{w_{X_{t}(x)}^{1}, \ldots, w_{X_{t}(x)}^{r}\right\}
$$

ii) there are Lebesgue-integrable functions $a_{i j}: J \longrightarrow R$ such that for each $t \in J$ and $i=1, \ldots, r$

$$
L_{X}\left(w^{i}\right)\left(X_{t}(x)\right)=\Sigma_{j=1}^{r} a_{i j}(t) w_{X_{t}(x)}^{j}
$$

Motivated by the co-distributions defined in relation to the problem of the observability of control systems, each distribution will be assumed to beas differentiable at $x$ for each $x \in M$.

Together with the concepts above, the following lemma is obtained.

Lemma 2.4. If $X \in X(M)$ preserves a co-distribution $\theta$ infinitesimally at $x$ along the parametrization $\delta=\left(w^{1}, \ldots, w^{r}\right)$ then, for each $\mathrm{t} \in \mathrm{J}$

$$
X_{t}^{*} \theta\left(X_{t}(x)\right)=\theta(x)
$$


Proof. . It is sufficient to show that for each $t \in d$ and for each $i=1, \ldots, r$

$$
r_{i}(l)=X_{i}(x) u_{\lambda_{1}(x)}^{i} \in O(x) .
$$

('ousider an application $\lambda \in T_{x}$ M such that $\theta(x) \subset \operatorname{her}(\lambda)$. Let us denote, for $i=1, \ldots, r$

$$
\gamma_{i}(t)=\lambda\left(\alpha_{i}(t)\right)
$$

Niter a derivation, we obtain:

$$
\begin{gathered}
\frac{d}{d t} a_{i}(t)=X_{i}^{*} L_{X}\left(w^{i}\right)\left(X_{t}(x)\right) \\
=X_{i}^{*}\left(\Sigma_{j=1}^{r} a_{i j}(t) w_{X_{i}\left(x_{i}\right)}\right) \\
=\Sigma_{j=1}^{r} a_{i j}(t) \alpha_{j} .
\end{gathered}
$$

Then

$$
\frac{d}{d t} \gamma_{i}(t)=\sum_{j=1}^{r} a_{i j}(t) \lambda\left(\alpha_{j}(t)\right)
$$

In particular if $\gamma$ denotes $\left(\gamma_{1}, \ldots, \gamma_{r}\right)$ as column vector, wo obtain

$$
\begin{gathered}
\gamma=\left(a_{i j}\right) \gamma \\
1 \leq i \leq r
\end{gathered}
$$

then $\gamma$ satisfies a hear differential cquation with Lebesque-integrable coeffecents, and thus the existence and uniquencse of the solutions are warranted. Now,

$$
a_{i}(0)=w_{\pi}^{i} \in \theta(w)
$$

and by the construction of $\lambda$, we obtain $\gamma(0)=0$ and consequently $\gamma \equiv 0$. Otherwist. for each $i=1, \ldots, r$

$$
\lambda\left(\alpha_{i}(t)\right)=0
$$

therefore for earh $i=1, \ldots, r \alpha_{i}(t) \in \theta(x)$. In fart, the reasoning above is valid for each $\lambda$ satisfying $\theta(x) \subseteq k \in r \lambda$

\section{Renuarks 2.5.}

Lemma 2.4 . establishes that if $X \in X(M)$ preserves $\theta$ infinitesimally at $x$ along $\delta$, then $\mathcal{X}$ preserves $\theta$ over the set

$$
\left\{X_{t}(x) \mid t \in J\right\}
$$

This situation is still insufficient since the parametrization $\delta$, explains only a "piece of trajectory through $x$ " and not a neightrorhood of point $x$. 
Definition 2.6. A parametrization $\delta=\left(w^{1}, \ldots, w^{r}\right)$ of $\theta$ is called exhaustive over a set $A \subset M$, if for each $y \in A$

$$
\theta(y)=\operatorname{Span}\left\{w_{y}^{1}, \ldots, w_{y}^{r}\right\}
$$

Now, we are really in a position to make the best of definition 2.3.

Lemma 2.7. Let us suppose that $\delta=\left(w^{1}, \ldots, w^{r}\right)$ is an exhaustive parametrization of $\theta$ over an open $V$ of $M$ and that $X \in X(M)$ preserves $\theta$ infinitesimally along $\delta$ at all points of $V$. Then, $X$ preserves $\theta$ in $V$.

Proof. . : We use a compactness argument. Let $T>0$ and y $y \in \bigcap_{i} \operatorname{dom}\left(X_{i}\right)$. Let us define the set $S$ by :

$$
S=\left\{t \in[0, T] X_{\sigma}^{*}\left(\theta\left(X_{\sigma}(y)\right)=\theta(y), \forall \sigma \in[0, T]\right\}\right.
$$

Lemma 2.4 states that $S$ is not empty. Let $s=S u p(S)$. Since $X$ preserves $\theta$ infinitesimally in $X_{s}(y) \in V$ along $\delta$, there exists $\varepsilon>0$ satisfying :

$$
-\varepsilon<t<\varepsilon \Longrightarrow X_{t}^{*} \theta\left(X_{t+s}(y)\right)=\theta\left(X_{s}(y)\right)
$$

Then, $s=T$ and $X$ preserves $\theta$

Let $F$ a transitive family of vector fields on $M$ and $\theta$ a co-distribution. The Lernmas in the above paragraph can be summarized as follows: if Lie's derivatives of exhaustive parametrizations of $\theta$ in the direction of the elements of $F$ can be written as linear combinations with the Lebesque-integrable coefficients of the parametrization, then $\mathrm{F}$ preserves $\theta$. We must study, therefore, co-distributions having this type of parametrizations.

\section{INTERGRABILITY}

We start with a new concept:

Definition 3.0. Let $F \subset X(M)$ and $\theta$ a co-distribution in $M . \theta$ is said to be $F$ involutive if

$$
L_{X}(w) \in \theta, \forall X \in F, \forall w \in \theta \square
$$

\section{Renuarks 3.1.}

1. If $F$ preserves $D$, then $\theta$ is $F$-involutive. In particular, $\nabla$ is $D_{\Sigma}$-involutive In fact, 


$$
\nabla=\operatorname{Span}\left\{d\left(h_{i} \circ \phi\right) \mid \phi \in G_{\Sigma}, i=1, \ldots, s\right\}
$$

and, if $\beta \in G_{\Sigma}$ and $d\left(h_{i} \circ \phi\right) \in \nabla$, we have:

$$
\beta^{*}\left(d\left(h_{i} \circ \phi\right)\right)=d\left(h_{i} \circ(\phi \circ \beta)\right)
$$

Since $\phi \circ \beta \in G_{\Sigma}$ it follows that $\beta^{*}\left(d\left(h_{i} \circ \phi\right) \in \nabla\right.$ for each $i=1, \ldots, s$. Consequently, $F=D_{\Sigma}$ preserves $\nabla$. In fact, the elements that generate $\nabla$ form exhaustive parametrizations of this co-distribution over opens sets of $M$.

2. From the definition of $\Lambda$ itself, we conclude that this co-distribution is $D_{\Sigma}$ -involutive.

3. $\nabla^{+}$is $D_{\Sigma}$-involutive, however, as seen before, $\nabla^{+}$may not be preserved by $D_{\Sigma}$.

Theorem 3.2. Let $F$ a transitive family over $M$ and $\theta$ a co-distribution in $M$. Then, $F$ preserves $\theta \Leftrightarrow \theta$ is regular and $F$-involutive.

Proof: Suppose that $\theta$ is regular and $F$-involutive and let $x \in M$ and $\delta=$ $\left(w^{1}, \ldots, w^{r}\right)$ a parametrization of $\theta$ centered in $x$ (in a neighborhood of $x$ ). $\theta$ is regular and since the 1 -forms $w^{j}, j=1, \ldots, r$ are continuous, there must be a neighborhood $V_{0}$ of $x$ such that

$$
\theta(y)=\operatorname{Span}\left\{w_{y}^{1}, \ldots, w_{y}^{r}\right\}, \forall y \in V_{o}
$$

(The argument used here is of the following type: if a continuous function is not null in a specific point, then it is not null in a certain neighborhood of the point considered).

Consequently, $\delta$ is an exhaustive parametrization of $\theta$ over $V_{o}$. By hypothesis $\theta$ is $F$-involutive and then

$$
\forall w \in \theta, \forall X \in F, L_{X}(w) \in \theta
$$

Thus, for $y \in V_{o}, i=1, \ldots, r$ we have:

$$
\left.L_{X}\left(w^{i}\right)\right|_{y}=\sum_{j=1}^{r} a_{i j} w_{y}^{j} .
$$


As $\left\{w^{1}, \ldots, w^{r}\right\}$ can be considered as a linearly independent set in $V_{o}$, we conclude that the functions $a_{i j}: V_{o} \longrightarrow \mathbb{R}$ are determined.

Now, if we prove that these functions are Lebesque-integrable, then since the reasoning above is independent from the point $y \in V_{o}^{r}$ and $X \in F$, Lemma 2.7 applies and hence $F$ would preserve $\theta$ in $V_{0}$. As the exhaustive parametrizations of $\theta$ cover $M$ thoroughly, the result would be proved. It is possible to prove something stronger: each $a_{i j}$ is a differentiable function.

For each $y \in V_{o}$ we considerer the linear mapping

$$
\delta(y): \mathbb{R}^{r} \longrightarrow \theta(y) \subset T_{y}^{*} M
$$

defined by

$$
\delta(y)\left(t_{1}, \ldots, t_{r}\right)=\sum_{j=1}^{r} t_{j} w_{y}^{j} .
$$

Then, $\delta$ is differentiable in $V_{o}$ and $\delta(y)$ has a range $r$. Let us also observe that

$$
\left.L_{X}\left(w_{i}\right)\right|_{y}=\delta(y)\left(a_{i 1}(y), \ldots, a_{i r}(y)\right)=\delta(y)\left(a_{i}(y)\right)
$$

is differentiable in $V_{0}$. Due to arguments on range, there are matrices $P(y) \in$ $\operatorname{Cr}_{r}(R), Q(y) \in M_{(m-y) x y}(\mathrm{IR})$, (where $m$, is of course the dimension of $M$ and hence the dimension of the co-tangent space $\left.T_{y}^{*} M\right)$, such that

$$
\delta(y)=\left(\begin{array}{l}
P(y) \\
Q(y)
\end{array}\right)
$$

The differentiability of $P(\cdot)$ is warranted by the differentiability of $\delta(\cdot)$. As for each $y \in V_{o}, P(y)^{-1}$ exist, we conclude that $P^{-1}(\cdot)$ is also differentiable. Now

$$
a_{i}(y)=\left(P(y)^{-1} O\right)\left(\begin{array}{c}
P(y) \\
Q(y)
\end{array}\right) a_{i}(y)=\left.\left(P(y)^{-1} O\right) L_{X}\left(w^{i}\right)\right|_{y}
$$

So, the applications $a_{i}=\left(a_{i 1}, \ldots, a_{i r}\right)$ are differentiable in a neighborhood of $x$. Since the reciprocal was already proved, the proof of the theorem is finished

Extensions of Theorem 3.2.

1. The differentiability of the matrix $\left(a_{i j}\right)$ in the above theorem is a condition quite stronger than the restriction Lebesque-integrable. When explaining this situation, the concept below comes up naturally.

Definition 3.3. $\theta$ is said to be $F$-weakly involutive, if for each $x \in M$, there is a neighborhood $U$ of $x$ and an exhaustive parametrization $\delta$ in $U$, such that, each $X \in F$ preserves $\theta$ infinitesimally in $x$ along $\delta$, with Lebesque-integrable coefficients

Theorem 3.4. Let $F$ a transitive family of vector fields over $M$ and $\theta$ an $F$ weakly-involutive co-distribution. Then $F$ preserves $\theta$.

Proof : From the hypothesis we deduce that $\theta$ is a regular co-distribution and the rest of the proof is contained in the proof of the theorem 3.2 
2. In lemma 2.4 the curves considered were

$$
\alpha_{j}(t)=X_{t}^{*}\left(w^{j}\right)\left(X_{t}(x)\right)
$$

The proof of this lemma results from the fact that $\beta=\lambda(\alpha)$ satisfies a linear differential equation with Lebesque-integrable coefficients and with initial condition $\beta(0)=0$. Let us consider again the definition $2.3(3)$ but this time with restrictions on derivatives of highest order, that is, condition (ii) is replaced by

$$
L_{X}^{(s)}\left(w^{i}\right)\left(X_{t}(x)\right)=\sum_{j=1}^{r} a_{i j}(t) w_{X_{t}(x)}^{j}
$$

with $a_{i j}$ Lebesque-integrable and $s \in N$.

Since,

$$
\alpha_{i}^{(s)}(t)=X_{t}^{*} L_{X}^{(s)}\left(w^{i}\right) X_{t}(s)
$$

then,

$$
\beta_{i}^{(s)}(t)=\sum_{j=1}^{r} a_{i j}(t) \cdot \beta_{j}(t), \quad 1 \leq i \leq r
$$

however, this equation is equivalent to the system

$$
\begin{gathered}
\beta^{(1)}(t)=\gamma_{1}(t) \\
\gamma^{(1)}(t)=\gamma_{2}(t) \\
\cdot \\
\gamma_{s-1}^{(1)}(t)=\left(a_{i j}\right) \beta(t) .
\end{gathered}
$$

If we assume that $L_{X}^{(p)}\left(w^{i}\right)(x) \in \theta(x), \quad s \geq p \geq 1$, that is, if $\gamma_{l}=0$ for $s-1 \geq l \geq 1$ we will have:

$\beta(s)$ satisfies a linear differential equation with Lebesque- integrable coefficients and $\beta^{(s)}(0)=0$. Due to the uniqueness of the solution we conclude that $\beta^{(s)} \equiv 0$. Inductively, we obtain

$$
\beta^{(p)} \equiv 0 \text { for } s \geq p \geq 1
$$

Then, $\beta \equiv 0$ and again the conclusion of the lemma 2.4 will be valid.

Definition 3.5. An exhaustive parametrization $\delta=\left(w^{1}, \ldots, w^{r}\right)$ of $\theta$ in $U$ is said to be $\mathrm{F}$-weakly-involutive of order $n \in N$, if for each $X \in F$, there are integers $l_{1}, \ldots, l_{r} \in\{1, \ldots, n\}$ such that for each $p \in\{1, \ldots, n\}$ and each $i=1, \ldots, r$

i) $L_{X}^{(p)}\left(w^{i}\right)(y) \in \theta(y), \quad \forall y \in U$ 
ii) The equation

$$
\delta(y) a_{i}(y)=L_{X}^{(l i)}\left(w^{i}\right)(y)
$$

admits solution with $a_{i}: l^{\prime} \longrightarrow$ IR Lebesque-integrable

Theorem 3.6. Let $F$ a transitive family of vector fields on $M, \theta$ a co-distribution and $n \in N$. If the domains of the parametrizations $F$-weakly-involutive of order $n$ of cover $M$, then $F$ preserves $\theta$.

Proof : It comes directly from the comment above and the definition 3.5 itself

Let $M$ a manifold, $X$ a vector field and $w$ a 1 -form, defined over $M$. Matsuda's condition [6] is transposed to our context as follows:

The series

$$
\sum_{k=0}^{\infty}(-1)^{k} \frac{t^{k}}{k !} L_{X}^{(k)}(w)(x)
$$

is of class $C^{1}$ in a neighborhood $U_{o}$ of $(0, x) \in \mathbb{I R} \times M$ and the derivatives may be computed by differentiating the series term by term.

With this idea, we get the following result.

Theorem 3.7. Let $F$ a transitive family of vector fields on $M$ and $\theta$ an $F$ involutive co-distribution. If the domains of the exhaustive parametrizations of $\theta$ that satisfy Matsuda's condition in relation to $F$ cover $M$, then $F$ preserves $\theta$.

Proof : Let $\delta=\left(w^{1}, \ldots, w^{r}\right)$ be an exhaustive parametrization of $\theta$ in a neighborhood $U$ of $x$. Let us define for $j=1, \ldots, r$

$$
\begin{gathered}
\alpha_{j}(t, \tau)=\sum_{k=0}^{\infty}(-1)^{k} \frac{t^{k}}{k !}\left(w^{j}\right)\left(X_{\tau}(x)\right) \\
\beta_{j}(t, \tau)=X_{\tau}^{*} \alpha_{j}(t, \tau) .
\end{gathered}
$$

Since $\theta$ is $F$-involutive, we can see that $\alpha_{j}(t, \tau) \in \theta\left(X_{j}(x)\right)$ By taking partial derivative, we get

$$
\begin{gathered}
\frac{\partial}{\partial t} \beta_{j}=\sum_{k=1}^{\infty}(-1)^{k} \frac{t^{k-1}}{(k-1) !} X^{*} \tau L_{X}(k)\left(w^{j}\right)\left(X_{\tau}(x)\right) \\
\frac{\partial}{\partial t} \beta_{j}=\sum_{k=0}^{\infty}(-1)^{k} \frac{t^{k}}{k !} X_{\tau}^{*} L_{X}^{(k+1)}\left(w^{j}\right)\left(X_{\tau}(x)\right)
\end{gathered}
$$

In particular, for each $j=1, \ldots, r, \frac{\partial}{\partial t} \beta j+\frac{\partial}{\partial \tau} \beta j=0$.

Since $\beta j(0.0)=w_{x}^{j}$ then $\beta_{j}(t, t)=w_{x}^{j}, \forall t$. Thus $X_{-\tau}^{*} w_{x}^{j}=\alpha j(t, t) \in \theta\left(X_{\tau}(x)\right)$ and then $\Sigma$ preserves $\theta$ 
Let $X$ and $w$ be analytical sections of the bundles $T M$ and $T^{*} M$ respectively. To study the curve

$$
\alpha(t)=X_{t}^{*} w_{X_{t}}(x)
$$

locally, it is sufficient to know the successive Lie's derivatives of the 1-form $w$ with respect to the vector field $X$, at one point.

Theorem 3.8. Suppose that $F$ is a transitive family of vector fields and $\theta$ is an analytical $F$-involutive co-distribution over $M$, such that the exhaustive analytical parametrization of $\theta$ cover $M$. Then $F$ preserves $\theta$.

Proof. Let $x \in M$ and $U$ a neighborhood of $x$ and the domain of an analytical exhanstive parametrization $\delta \pm\left(w^{1}, \ldots, w^{r}\right)$. For each $X \in F$ and $i=1, \ldots, r$

$$
\alpha_{i}(t)=X_{t}^{*} w_{X_{t}}^{i}(x)
$$

defines an analytical curve over $T^{*} M$. Since $\theta$ is $F$-involutive, we get

$$
\alpha_{i}^{(k)}(0)=L_{X}^{(k)}\left(w^{i}\right)(x) \in \theta(x) .
$$

From the analyticity, we conclude the existence of $\varepsilon>0:|t|<\varepsilon \Leftrightarrow \alpha_{i}(t) \in \theta(x)$ ,$\delta$ is exhaustive over $U$ and thus, by using the same type of argument utilized in lermma 2.7 , we see that $X$ and then $F$ preserves $\theta$ in $U$

Theorem 3.8. is not valid in the $C^{\infty}$ case. Indeed, consider the system $\Sigma$ defined by $M=\mathbb{R}^{2}, D=\left\{\frac{\partial}{\partial x}, \frac{\partial}{\partial y}\right\}$ and as in example $2.0(2)$.

Then

$$
\Lambda=\operatorname{Span}\left\{f^{(k)} d x\right\}
$$

is $D$-involutive and $\delta=f d x$ is a global $C^{\infty}$ parametrization of $\Lambda$. However, $D$ cannot preserve this co-distribution because of the fact that $\Lambda$ is not integrable in any point of the $y$ axis. In fact, the dimension of $\Lambda$ varies along any integral curve of the vector field $X$.

This example also shows that in the $C^{\infty}$ case, $\Lambda$ may not integrable, even though the vector fields of $D$ are analytical and complete and the system $\Sigma$ is symmetric and controllable. Indeed, the conclusion of the non-integrability of $\Lambda$ remains invariable if we consider

$$
D=\left\{ \pm \frac{\partial}{\partial x}, \pm \frac{\partial}{\partial y}\right\}
$$

instead of $D$ as above

Stefan's condition [8] is seen in the following concept.

Definition 3.9. Let $A \subset M$, a $A$ co-distribution $\theta$ is said to be locally analytic along $A$ if : for each $x \in A$ and $v \in \theta(x)$ there is an analytic form $w$ defined in a neighborhood of $A, w \in \theta$ and such that $w_{x}=v$

We observe that in the previous example there are exhaustive analytic parametrizations of $\Lambda$ for $x>0$ and for $x<0$. However, it is not possible "to connect" there parametrizations via analytic continuation. In fact, $\Lambda$ is not locally analytic along any piece of trajectory of the vector field $X=\frac{\partial}{\partial x}$ such that the set 


$$
A=\left\{X_{t} \mid t \in J\right\}
$$

contains points with positive, and negative first coordinate. This is because, for $v=d x$ and $z=(x, y) \in A$ with $x>0$ there is not $u \in \Lambda$ such that $w_{z}=v$. In fact, each

$$
w=a(x, y) d x+b(x, y) d y \in \Lambda
$$

is such that $a(x, y)$ is null over an open subset of $\operatorname{IR}^{2}$ so that $a \equiv 0$ in the plane, hecause of analyticity.

Theorem 3.10. Let $F$ a transitive family of vector fields on $M$ and $\theta$ an analytic $F$-involutive co-distribution. Then, $F$ preserves $\theta \Leftrightarrow$ for each $X \in F, \theta$ satisfies : if $x \in \operatorname{dom}(X)$ there exist $\varepsilon>0$ such that $\theta$ is locally analytic along the set

$$
A(X, x, \varepsilon)=\left\{X_{\ell}(x) \quad|| t \mid<\varepsilon\right\} .
$$

Proof: Let $X \in F$ and $x \in \operatorname{dom}(X)$. There is an analytic parametrization $\delta=$ $\left(w^{1}, \ldots \ldots, w^{r}\right)$ of $\theta$ centered in $x$ and defined in $U$. Let $\varepsilon>0$ so that

$$
-\varepsilon<t<\varepsilon \Rightarrow X_{t}(x) \in U .
$$

Let $y=X_{t_{o}}(x) \in A(X, x, \varepsilon)$ and consider $v \in \theta(y)$.

Since $X$ preserves $\theta$, there are $u_{1}, \ldots, u_{r}$ such that

$$
X_{t_{o}}^{*}(v)=\sum_{j=1}^{r} u_{j} W_{x}^{j}
$$

Then

$$
w=X_{-t_{0}}^{*}\left(\sum_{j=1}^{r} u_{j} W^{j}\right)
$$

is an analytic form defined in a neighborhood of $A(X, x, \varepsilon)$ and $w_{x}=v$.

Conversely let $X \in F$ and $x \in \operatorname{dom}(X)$. There exist $\varepsilon>0$ such that $\theta$ is locally analytic along $A(X, x, \varepsilon)$. We must prove that

$$
|t|<\varepsilon \Rightarrow X_{t}^{*} \theta\left(X_{t}(x)\right) \subset \theta(x)
$$

For each $v \in \theta\left(X_{\tau}(x)\right)$, there exists $w \in \theta$ with $v=W_{X_{t}}(x)$.

Let us define the analytic curve

$$
\alpha(s)=X_{s}^{*} W_{X_{s}}(x)
$$

By deriving $\alpha$ and evaluating at $s=0$, we obtain: $\alpha(s) \in \theta(x)$ for each $s \in \operatorname{dom}()$.

Thus, $X_{t}^{*} W_{X_{t}}(x) \in \theta(x)$ and consequently due to the arbitrarity of $v$ we conclude that

$$
X_{t}^{*} \theta\left(X_{t}(x)\right) \subset \theta(x), \text { for }|t|<\varepsilon
$$

The proof follows then in the same way as lemma 2.7 .

This result generalizes theorem 3.8. In fact, in this case what is necessary is the exhaustivity of $\theta$ along the trajectories of the vector fields of $F$. 


\section{A. $\Gamma^{+}$AND $\nabla$}

Somf facts about $\Lambda, \nabla^{+}$and $\nabla$ are deduced. Other results may be found in, [2]. Proposition 4.1. Let $\Sigma=\left(M, D, \mathrm{IR}^{\mathrm{5}}, h\right)$ a control system. If the dimension of 1 is constant, then

$$
\Lambda=\nabla^{+}=\nabla
$$

Proof : is regular and $D$-involutive by definition, so the theorem 3.2 . is applied and then $D$ preserves $\Lambda$. Let $i=1, \ldots, s$ and $X \in D$. For each possible $t \in R$, the $D$ - invariance of $\Lambda$ ensures that

$$
d\left(h_{i} \circ X_{t}\right)=X_{t}^{*}\left(d h_{i}(x)\right) \in \Lambda(x), \quad \forall x \in M .
$$

The same reasoning is valid for any possible finite combination of elements in $D$. That is, if

$$
\rho=X_{t_{1}} \ldots X_{t_{k}} \in G_{\Sigma}
$$

then, for each $X \in M$

$$
d\left(h_{i} \circ \rho\right)=\rho^{*} d h_{i}(x) \in \Lambda(x) .
$$

Thus, $\nabla(x) \subset \Lambda(x), \forall x \in M$ and the result comes from the fact that $\Lambda \subset$ $\Gamma^{+} \subset \nabla$, due to the construction itself of those objects

In [1] was proved the following proposition

Let $\Sigma=\left(M, D, \mathbb{R}^{\mathrm{S}}, h\right)$ a transitive control system then,

$$
\operatorname{dim}\left(\nabla^{+}\right) \text {constant } \Leftrightarrow \nabla^{+}=\nabla
$$

In particular, we obtain:

Corollary 4.2. Let $\Sigma$ a transitive control system. Then

a) $\nabla^{+}=\nabla \Leftrightarrow \nabla^{+}$is integrable

b) $\Sigma$ controllable $\Rightarrow \nabla^{+}=\nabla$.

c) $\Sigma$ analytic $\Rightarrow \Lambda=\nabla^{+}=\nabla$.

\section{Proof :}

a) Since $\Sigma$ is transitive, we deduce that $\nabla=\nabla^{+}$is integrable. Conversely, the integrability of $\nabla^{+}$implies that this co-distribution is regular and consequently $\Gamma^{+}=\nabla$ by the above result

b) If $\Sigma$ is controllable, then given $x, y \in M, \exists \rho \in S_{\Sigma}$ such that $y=\rho(x)$. Since $\nabla^{+}$is $S_{\Sigma}$-invariant, the dimension of $\nabla^{+}$is independent from the states in $M$, thus $\Gamma^{+}=\nabla$.

c) Inder these conditions $\Lambda$ is integrable and therefore regular. The result comes directly from proposition 4.1 .

\section{References}

[1] Ayala. V. and San Martin, L., Minimal realizations under controllability, Systems Control Letters

16 (1991) 289-29:3. 
[2] Ayala, V., Sobre a Observabilidade de Sistemas de Controle, Doutor em Ciencias, Thesis, Universidade Estadual de Campinas, Brasil, 1988.

[3] Basto Concalvez, J. Nonlinear observability and duality, Systems Control Letters 4 (1984) 97-101.

[4] Hermann, R. and Krener, A. Nonlinear controllability and observability, IEEE Trans. Automat. Control 22(5) (1977) 728-740.

[5] Lobry,C., Controlabilite des systemes non lineaires, SIAM J. Control 8 (1970) 573-605.

[6] Matsuda, M., An integration theorem for completely integrable systems with singularities, Osaka J. Math. 5 (1968), 279-283.

[7] Stefan, P., Accesible sets, orbits and foliations with singularities, Proc. London Math. Soc. 29 (1974) 699-713.

[8] Stefan, P., Integrability of Systems of Vector Fields, J. London Math. Vol. 2, $21(1980)$.

[9] Sussmann, H., Orbits of families of vector fields and integrability of distributions, Trans. Amer. Math. Soc. 180 (1973) 171-188.

[10] Warner, F., Foundations of Differentiable Manifolds and Lie Groups, Scott, Foresman and Company, Glenview Illinois, 1971.

Received: November 2, 1993

Víctor Ayala Bravo

Departamento de Matemática

Facultad de Ciencias

Universidad Católica del Norte

Casilla 1280, Antofagasta-Chile. 